Supplementary Information

\title{
Liposomal surface-loading of water-soluble cationic iron(III) porphyrins as anticancer drugs
}

Makoto Yuasa,* Kenichi Oyaizu, Aiko Horiuchi, Akihiko Ogata, Tomomi Hatsugai, Aritomo Yamaguchi, and Hiroyoshi Kawakami

Department of Pure \& Applied Chemistry, Faculty of Science and Technology, Tokyo University of Science, Noda 278-8510, Japan, Institute of Colloid and Interface Science, Tokyo University of Science, Tokyo 162-8601, Japan, and Department of Applied Chemistry, Tokyo Metropolitan University, Tokyo 192-0397, Japan

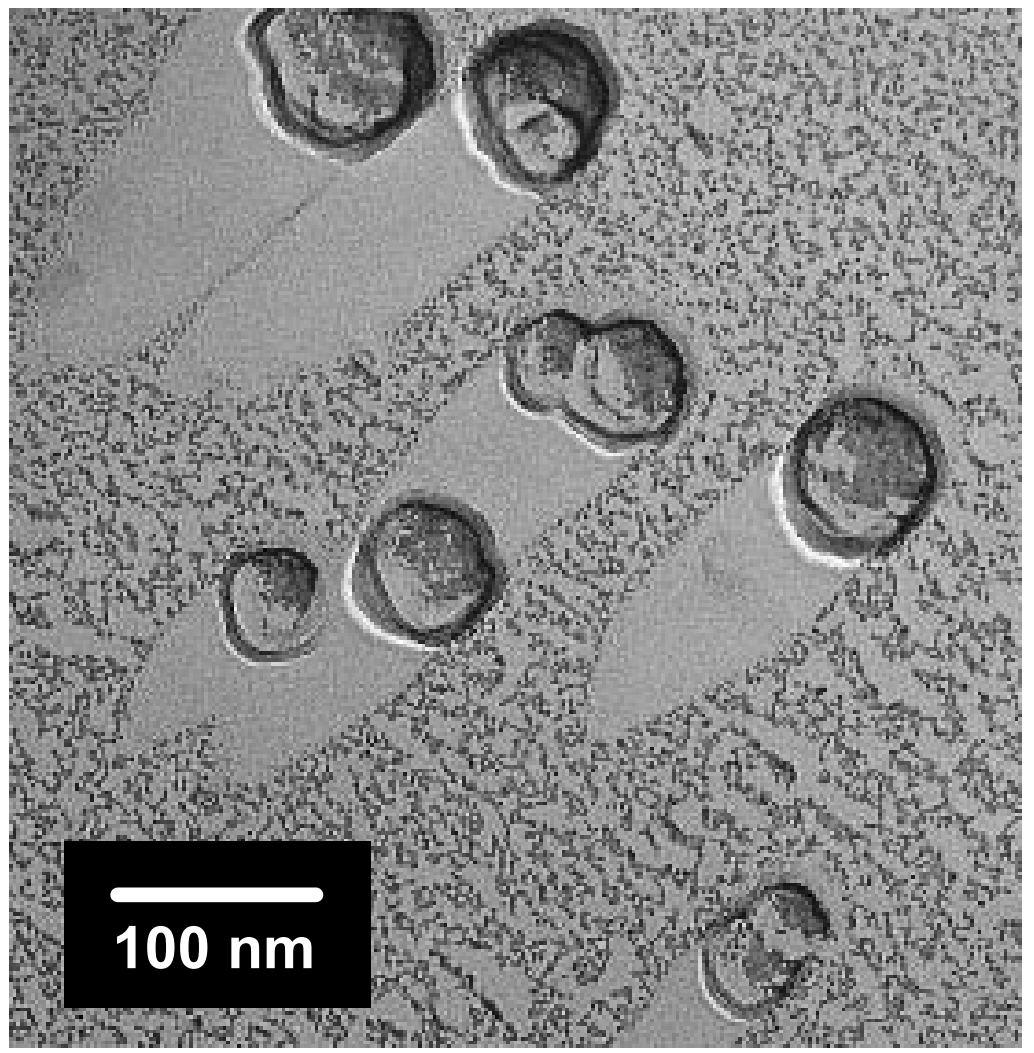

Fig. S1 Magnified TEM picture of $\mathbf{1}$. 


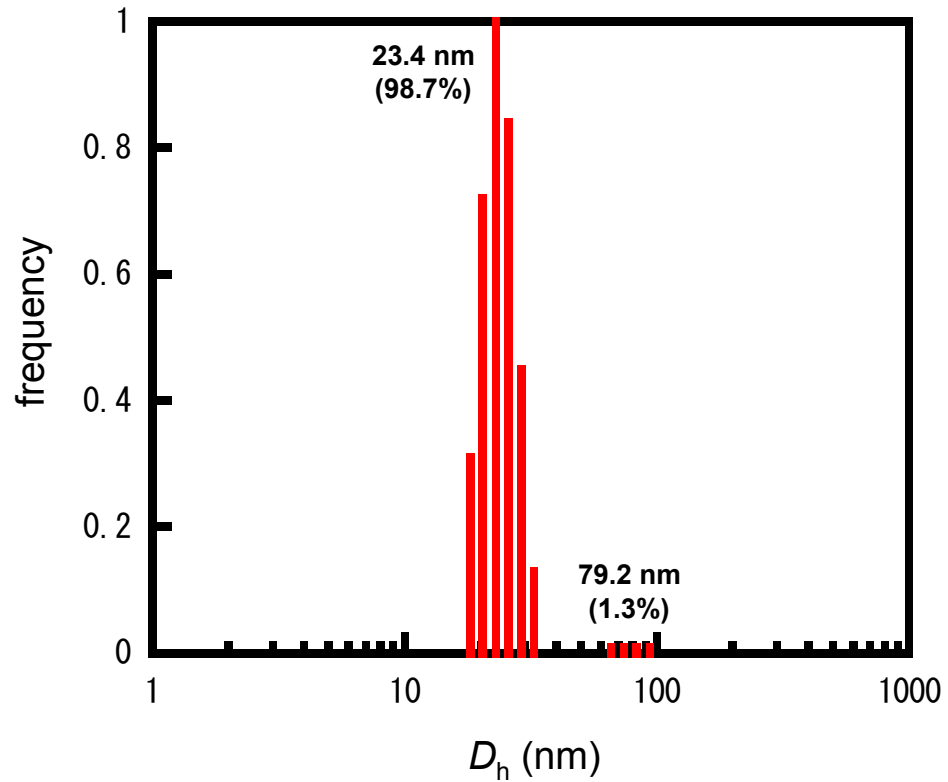

Fig. S2 A dynamic light Scattering histogram showing the hydrodynamic diameter $\left(D_{\mathrm{h}}\right)$ of 1 . Detection angle: $90^{\circ}$. 